\begin{tabular}{|c|l|}
\hline Title & Localization of exogenous DNA to mitochondria in skeletal muscle following hydrodynamic limb vein injection \\
\hline Author(s) & Y asuzaki, Y ukari; Y amada, Y uma; Kanefuji, Tsutomu; Harashima, Hidey oshi \\
\hline Citation & $\begin{array}{l}\text { Journal of controlled release, 172(3), 805-811 } \\
\text { https://doi.org/10.1016/.jconrel.2013.09.029 }\end{array}$ \\
\hline Issue Date & 2013 12-28 \\
\hline Doc URL & http://hdl.handle.net/2115/57267 \\
\hline Type & article(author version) \\
\hline File Information & Wos_64052_Y amada.pdf \\
\hline
\end{tabular}

Instructions for use 


\section{Localization of exogenous DNA to mitochondria in skeletal muscle following hydrodynamic limb vein injection}

Yukari Yasuzaki ${ }^{\mathrm{a}, \mathrm{c}}$, Yuma Yamada ${ }^{\mathrm{a}, \mathrm{c}}$, Tsutomu Kanefuji ${ }^{\mathrm{b}}$ and Hideyoshi Harashima ${ }^{\mathrm{a}, *}$

${ }^{a}$ Laboratory for Molecular Design of Pharmaceutics, Faculty of Pharmaceutical Sciences, Hokkaido University, Sapporo, Japan

${ }^{\mathrm{b}}$ Division of Gastroenterology and Hepatology, Niigata University, Niigata, Japan.

${ }^{\mathrm{c}}$ These authors contributed equally as first author

*To whom correspondence should be addressed:

Tel +81-11-706-3919 Fax +81-11-706-4879 E-mail harasima@pharm.hokudai.ac.jp 
ABSTRACT.

Mitochondrial genetic disorders are a major cause of mitochondrial diseases. It is therefore likely that mitochondrial gene therapy will be useful for the treatment of such diseases. Here, we report on the possibility of mitochondrial gene delivery in skeletal muscle using hydrodynamic limb vein (HLV) injection. The HLV injection procedure, a useful method for transgene expression in skeletal muscle, involves the rapid injection of a large volume of naked plasmid DNA (pDNA) into the distal vein of a limb. We hypothesized that the technique could be used to deliver pDNA not only to nuclei but also mitochondria, since cytosolic pDNA that is internalized by the method may be able to overcome mitochondrial membrane. We determined if pDNA could be delivered to myofibrillar mitochondria by HLV injection by PCR analysis. Mitochondrial toxicity assays showed that the HLV injection had no influence on mitochondrial function. These findings indicate that HLV injection promises to be a useful technique for in vivo mitochondrial gene delivery.

\title{
Keywords
}

Mitochondria disease, mitochondrial gene delivery, hydrodynamic limb vein injection, mitochondrial gene therapy.

\begin{abstract}
Abbreviations
Ch, channel; CLSM, confocal laser scanning microscopy; COX, cytochrome c oxidase; DAPI, 4', 6Diamidino-2-phenylindole; FCCP, carbonyl cyanide 4-(trifluoromethoxy)phenylhydrazone; FISH, fluorescent in situ hybridization; GAPDH, Glyceraldehyde 3-phosphate dehydrogenase; HLV, hydrodynamic limb vein; MELAS, Mitochondrial myopathy, encephalopathy, lactic acidosis and stroke-like episodes; MERRF, Myoclonic epilepsy and ragged-red fiber disease; MIB, mitochondrial isolation buffer; MTDR, MitoTracker Deep Red 633; PBS (-), phosphate-buffered saline; pDNA, plasmid DNA; q-PCR, quantitative real-time PCR; RLU, relative light units; TMRM, Tetramethylrhodamine.
\end{abstract}




\section{INTRODUCTION}

Mitochondrial dysfunction has been implicated in a variety of human diseases [1-3]. It is noteworthy that such disorders are mainly associated with tissues that have high energy requirements, such as the brain, heart, muscle and liver. It is now well accepted that mutations and defects in the mitochondrial genome form the basis of these diseases [4-7]. Therefore, mitochondrial gene therapy and diagnosis would be expected to have substantial medical benefits. However, their utility has not yet been realized because mitochondrial gene delivery technology is the bottleneck. Even through in vitro experiments have been reported, actual studies of mitochondrial gene delivery are very few [8-11]. The use of a variety of applications for nuclear gene delivery in vitro/in vivo have been reported [11-16], in attempts to accelerate progress in the field of nuclear gene therapy. Thus, successful mitochondrial gene transfer would largely contribute to mitochondrial gene therapy.

Skeletal muscle represents an attractive target tissue for mitochondrial gene therapy as well as nuclear gene therapy, because mitochondrial diseases, including Mitochondrial myopathy, encephalopathy, lactic acidosis and stroke-like episodes (MELAS) [5] and Myoclonic epilepsy and ragged-red fiber disease (MERRF) [7] are largely associated with mitochondrial genomic dysfunction in skeletal muscle. Vascular delivery procedures have recently been used to deliver plasmid DNA (pDNA) to the skeletal muscle of rodents and nonhuman primates by hydrodynamic limb vein (HLV) injection [17-20]. The hydrodynamic injection method originally was reported by Liu et al. [21] and Zhang et al. [22], and was used to achieve effective nuclear transgene expression in hepatocytes in mice by the rapid injection of large volumes of naked pDNA into the tail vein. Recently, Yan et al. reported on a facile hydrodynamic injection method via the retro-orbital sinus [23].

In the HLV injection procedure, a tourniquet is used to limit the delivery area to one limb per injection and naked pDNA is rapidly injected into the vein in the anterograde direction [20]. A sufficient volume of saline is used to facilitate extravasation of the pDNA from the vasculature and into the muscle tissue through multiple physical barriers. It has been suggested that hydrodynamic force could induce the transient opening of cellular membrane to permit pDNA to be internalized into cells, the subsequent localization of pDNA into the nucleus may be achieved via some mechanisms such as active nuclear import, cell division or transient opening of nuclear membrane, etc. More recently, it has been reported that the HLV injection of condensed pDNA was even more effective than naked pDNA in achieving nuclear transgene expression in skeletal muscle [24, 25] [26]. Based on these reports, we hypothesized that the HLV injection technique might be useful for delivering pDNA into, not only the nucleus, but also mitochondria. 
The purpose of this study was to validate the possibility of mitochondrial DNA delivery in skeletal muscle of rats by HLV injection (Fig. 1a). We first verified, using PCR analysis, that the HLV injection technique could be used to deliver naked pDNA into myofibrillar mitochondria.

We also investigated the effects of injection volume and dose of pDNA on mitochondrial delivery by HLV injection. A combination immunostaining of mitochondrial proteins and a

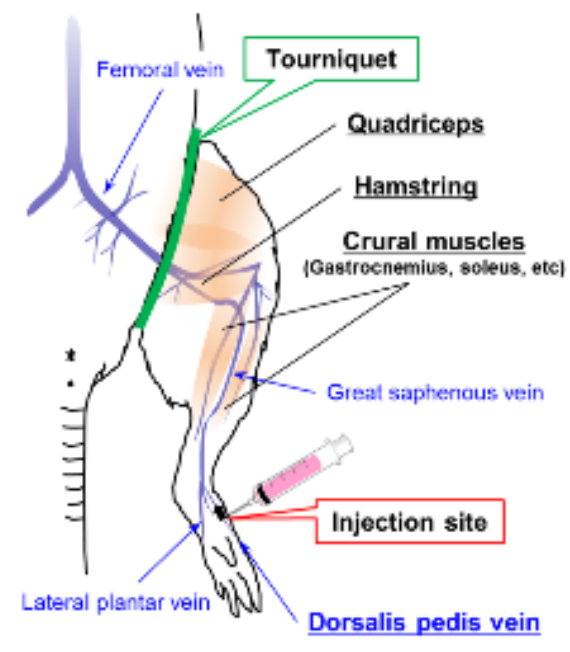

b

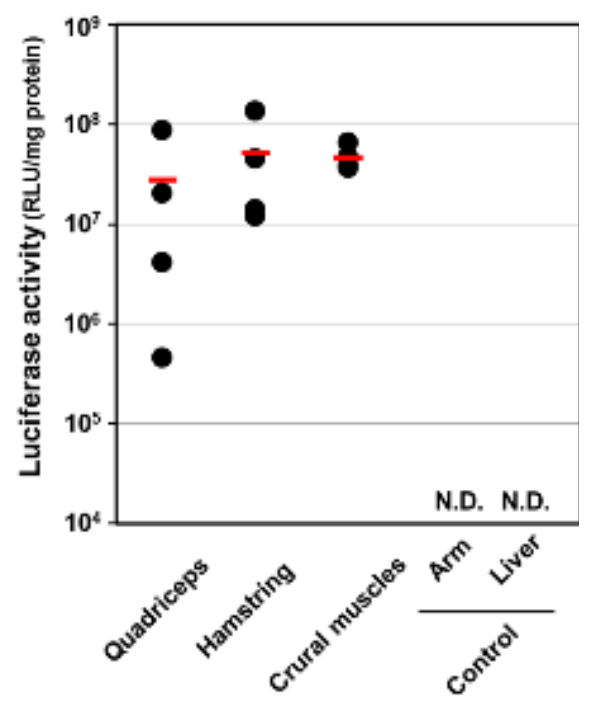

Fig. 1. Schematic images of gene delivery to skeletal muscle by HLV injection and evaluation of nuclear transgene expression in part of skeletal muscles. (a) Schematic diagram of HLV injection of pDNA into distal hind limb of rats. pDNA was intravenously injected into the dorsalis pedis vein under the conditions that restrict blood flow with a tourniquet. At $24 \mathrm{hr}$ postinjection, rats were sacrificed, the limb muscles were harvested and separated into three groups (quadriceps, hamstring, crural muscles). (b) Gene expression in part of skeletal muscles. Luciferase activities were measured at $24 \mathrm{hr}$ after HLV injection of pDNA coding luciferase. Bars represent the mean values $(n=4)$. Statistical analysis among Quadriceps, Hamstring and Crural muscles was performed by one-way ANOVA $(p=0.69)$. We also measured luciferase activities in the arm and liver as controls. N.D., not determined.

fluorescent in situ hybridization to detect pDNA (Immuno-FISH) permitted us to visualize the pDNA that was delivered to skeletal muscle tissue. Finally, we assessed the mitochondrial toxicity in skeletal muscle following HLV injection in terms of cytochrome c oxidase (COX) activity and mitochondrial membrane potentials.

\section{MATERIALS AND METHODS}

\section{Materials}

The pcDNA3.1 (+)-luc plasmid was constructed by inserting the firefly luciferase gene (Hind IIIXba I fragment) of the pGL3-Control plasmid (Promega, Madison, WI, USA) into the pcDNA3.1 (+) plasmid (Invitrogen, Carlsbad, CA, USA) pretreated with the same restriction enzymes. The luciferase gene in the pDNA is expressed under the control of the cytomegalovirus promoter. The pDNA was purified using an Endfree Plasmid Giga Kit (Qiagen GmbH, Hilden, Germany). Oligonucleotides were purchased from Sigma Genosys Japan (Ishikari, Japan) in purified form. Primary antibodies against COX 
IV, Lamin A + C or Glyceraldehyde 3-phosphate dehydrogenase (GAPDH) were purchased from Abcam (Cambridge, UK). 4', 6-Diamidino-2-phenylindole (DAPI) was obtained from Wako Pure Chemical Industries, Ltd. (Osaka, Japan). All other chemicals used were commercially available reagent-grade products.

\section{Experimental animals}

Female Wistar Hannover rats (7-9 weeks old) were purchased from Sankyo Labo Service (Sapporo, Japan). Rats with body weight in the range 150-180 g were used in all experiments. All animal protocols were approved by the institutional animal care and research advisory committee at the Faculty of Pharmaceutical Sciences, Hokkaido University, Sapporo, Japan.

\section{Hydrodynamic injection into the limb vein of rats}

Rats were anesthetized with a pentobarbital $(37.5 \mathrm{mg} / \mathrm{kg})$ solution via an intraperitoneal injection. Prior to each pDNA injection, a tourniquet was placed on the upper hind limb to restrict blood flow into and out of the hind limb. Basically, a suspension of naked pDNA ( $3 \mathrm{~mL})$ containing $184 \mu \mathrm{g}$ of pDNA was injected in $20 \mathrm{sec}$ from a distal site of dorsalis pedis vein. At $2 \mathrm{~min}$ after the injection, the tourniquet was released.

\section{Luciferase assay of muscle tissue after injection}

At $24 \mathrm{hr}$ postinjection, rats were sacrificed and the limb muscles were harvested and separated into three groups (quadriceps, hamstring, crural muscles). Each of the muscle tissues were minced with scissors and frozen in liquid nitrogen. Approximately $250 \mathrm{mg}$ samples of frozen muscle tissue were then homogenized using a PreCellys (bertin technologies, Montigny-le-Bretonneux, France) in $1 \mathrm{~mL}$ of lysis buffer (100 mM Tris-HCl, 2 mM EDTA, 0.1\% Triton X-100, pH 7.8). After centrifugation at 16,630g for $10 \min$ at $4^{\circ} \mathrm{C}$, a $20 \mu \mathrm{L}$ aliquot of the supernatant was used for a luciferase assay using the Luciferase Assay System (Promega, Madison, WI, USA). Protein concentrations were determined with a BCA protein assay kit (Pierce, Rockford, IL). Luciferase activities are expressed as relative light units (RLU) per mg of protein.

\section{Isolation of mitochondria from rat skeletal muscle}

At $24 \mathrm{hr}$ postinjection, the rats were sacrificed and the crural muscles were harvested and minced with scissors, and $1 \mathrm{~mL}$ of ice-cold mitochondrial isolation buffer [MIB: 250mM sucrose, $2 \mathrm{mMTris}-\mathrm{HCl}$, 
1 mM EDTA, pH 7.4] was then added. The suspension was homogenized using a PreCellys, and centrifuged at $800 \mathrm{~g}$ for $5 \mathrm{~min}$ at $4^{\circ} \mathrm{C}$. A $500 \mu \mathrm{L}$ of supernatant was transferred into ice-cold tubes containing $500 \mu \mathrm{L}$ of $\mathrm{MIB}$, and then centrifuged at $800 \mathrm{~g}$ for $5 \mathrm{~min}$ at $4{ }^{\circ} \mathrm{C}$. A $500 \mu \mathrm{L}$ of supernatant was transferred into ice-cold tubes containing $500 \mu \mathrm{L}$ of MIB, and the suspensions were centrifuged at 7,500g for $10 \mathrm{~min}$ at $4^{\circ} \mathrm{C}$. The pellets were washed with EDTA-free MIB, and the pellets were resuspended with EDTA-free MIB and the resulting suspensions were used as the mitochondria-enriched fraction. Purity of mitochondria-enriched fraction was confirmed by western blotting to detect organelle specific protein.

\section{Western blotting}

An equal volume of the sample was mixed with loading buffer [100 mM Tris- $\mathrm{HCl}$ (pH 6.8), 4\% SDS, 12\% 2-mercaptoethanol, 20\% glycerol, $0.05 \%$ bromophenol blue], and then subjected to $15 \%$ SDSPAGE. After electrophoresis, the proteins were electroblotted onto a Polyvinylidene Fluoride membrane (NIPPON Genetics Co., Ltd; Tokyo, Japan), and the membranes were then blocked with 5\% nonfat dry milk. Primary antibodies from mouse against the COX IV, Lamin A+C and GAPDH, as mitochondrial, nuclear and cytosolic fraction marker, were used at 1:1000, 1:500, and 1:10000, respectively. These proteins were further detected using secondary HRP-conjugated anti-mouse antibodies (GE Healthcare UK Ltd, Buckinghamshire, England) at a 1:1000 dilution. Blots were developed with Amersham ${ }^{\mathrm{TM}}$ ECL Plus Western Blotting Detection System (GE Healthcare), and immunoreactive bands were visualized using the LAS 4000 (Fujifilm, Tokyo, Japan).

\section{Detection of pDNA in mitochondria-enriched fraction after HLV injection.}

At $24 \mathrm{hr}$ postinjection, the rats were sacrificed, the crural muscles were harvested, and the mitochondria-enriched fraction was then obtained from the tissue. We also performed an intramuscular (IM) injection of naked pDNA at the quadriceps (184 $\mu \mathrm{g}$ of pDNA in $100 \mu \mathrm{L}$ solution per one injection). We extracted pDNA and mtDNA from the mitochondria-enriched fraction using SepaGene (Sanko Junyaku, Tokyo, Japan), and subjected to the PCR. The PCR reaction mixture contained, in a total volume of $20 \mu \mathrm{L}, 10 \mathrm{mM}$ of TrisHCl (pH 8.3), $1.5 \mathrm{mM}$ of $\mathrm{MgCl}_{2}, 50 \mathrm{mM}$ of KCl, $200 \mu \mathrm{M}$ of each one of the deoxynucleoside triphosphates, $0.2 \mu \mathrm{M}$ of primers Luc (+) and Luc (-) for pDNA detection or primers Rat D-loop (+) and Rat D-loop (-) for mtDNA (Table S1), and 10 ng of DNA obtained from the mitochondriaenriched fraction. After the addition of $0.5 \mathrm{U}$ of Taq DNA polymerase (Finnzymes PCR Reagents; Thermo Fisher Scientific, Waltham, MA, USA), the reaction mixture was first incubated at $95^{\circ} \mathrm{C}$ for $2 \mathrm{~m}$, then subjected to 30 cycles of $30 \mathrm{~s}$ at $95^{\circ} \mathrm{C}, 30 \mathrm{~s}$ at $60^{\circ} \mathrm{C}$ and $45 \mathrm{~s}$ at $72^{\circ} \mathrm{C}$, and finally to $5 \mathrm{~m}$ at $72^{\circ} \mathrm{C}$. Each $5 \mu \mathrm{L}$ of PCR products were subjected to electrophoresis in $2 \%$ agarose gel in TAE ( $40 \mathrm{mM}$ TriseHCl, 40 
$\mathrm{mM}$ acetic acid, $1 \mathrm{mM}$ EDTA, $\mathrm{pH} 8.0$ ) at $100 \mathrm{~V}$ for $30 \mathrm{~min}$. The DNA bands were visualized by UV after ethidium bromide staining.

\section{Quantification of pDNA in mitochondria-enriched fraction after injection}

At $24 \mathrm{hr}$ postinjection, rats were sacrificed and the crural muscles were harvested, and the mitochondria-enriched fraction was then obtained from the tissue. We first extracted mtDNA from the muscle tissue using GenElute Mammalian Genomic DNA Miniprep Kit (SIGMA-Aldrich Co.) to determine the copy numbers of mtDNA per mg muscle protein (mtDNAmuscle $[\mathrm{mtDNA}-\mathrm{mopy} / \mathrm{mg}$ muscle protein]). Copy numbers of mtDNA were estimated by quantitative real-time PCR (q-PCR) and concentrations of the muscle protein were measured using a BCA protein assay kit. We next extracted pDNA and mtDNA from the mitochondria-enriched fraction using SepaGene (Sanko Jun-yaku, Tokyo, Japan), and copy numbers of pDNA and mtDNA were measured by q-PCR to determine the amount of pDNA per mtDNA in mitochondria-enriched fraction (pDNAmt [fg pDNA/mtDNA-copy]). The amount of pDNA in mitochondria-enriched fraction of muscle tissues were calculated as follows:

Mitochondrial delivery of pDNA [fg pDNA/mg muscle protein] $=$ mtDNAmuscle [mtDNA-copy/mg muscle protein] X pDNAmt [fg pDNA/mtDNA-copy].

\section{DNase treatment of mitochondria-enriched fraction following HLV injection of pDNA}

At $24 \mathrm{hr}$ after the HLV injection of pDNA suspensions $(184 \mu \mathrm{g}, 3 \mathrm{~mL})$, the rats were sacrificed, the crural muscles were harvested, and a mitochondria-enriched fraction was then obtained from the tissue. The mitochondria-enriched fraction in a total volume of $38 \mu \mathrm{L}$ was incubated with $20 \mathrm{U}$ of Recombinant DNase I (RNase-free) [Takara Bio INC, Shiga, Japan] at $37^{\circ} \mathrm{C}$ for $30 \mathrm{~min}$, and $2 \mu \mathrm{L}$ of $0.5 \mathrm{M}$ EDTA (pH 8.0) was then added and the suspension was incubated at $80^{\circ} \mathrm{C}$ for $2 \mathrm{~min}$ to stop the reaction. A similar procedure was carried out, but without the DNase I treatment to produce a sample that had not been subjected to DNase I treatment. We next extracted pDNA and mtDNA from the mitochondria-enriched fraction, and the amount of pDNA and mtDNA were measured by q-PCR. Finally we determined the amount of pDNA per mtDNA in mitochondria-enriched fraction (ng pDNA/ mg mtDNA).

\section{Quantification of DNA by q-PCR}

q-PCR was performed using a 7500 Real Time PCR system (Applied Biosystems, Foster City, CA) with the following parameters: an initial denaturation at $95^{\circ} \mathrm{C}$ for $10 \mathrm{~min}$, followed by 40 cycles at $95^{\circ} \mathrm{C}$ for $15 \mathrm{sec}$ and $60^{\circ} \mathrm{C}$ for $1 \mathrm{~min}$. SYBR Green qPCR Mastermix (TOYOBO, Osaka, Japan) was used to indicate target gene amplification and the specificity of amplification was examined by automated dissociation stage analysis. A portion of the extracted DNA was analyzed for pDNA or mtDNA, using 
primers Luc (+) and Luc (-) and primers Rat D-loop (+) and Rat D-loop (-) [Table S1]. The copy number of pDNA or mtDNA was absolutely quantified using real time PCR.

\section{COX staining}

At $24 \mathrm{hr}$ postinjection, rats were sacrificed and the crural muscles containing the gastrocnemius/soleus were harvested, COX staining was performed on dried tissue sections. COX is the collective name for part of the oxidative respiratory chain of enzymes that are located exclusively in the mitochondria of cells. Briefly, 5- $\mu \mathrm{m}$ thick sections were attached on coverslips and placed in the incubating medium $\left(0.1 \mathrm{M} \mathrm{CH}_{3} \mathrm{COONa}, 2 \mathrm{mg} / \mathrm{mL}\right.$ di-amino benzidinetetrachloride (DAB), $0.1 \% \mathrm{MnCl}_{2}$, $0.1 \% \mathrm{H}_{2} \mathrm{O}_{2}, \mathrm{pH} 5.5$ ) for $1 \mathrm{hr}$ at $37^{\circ} \mathrm{C}$. The section was washed with deionized distilled $\mathrm{H}_{2} \mathrm{O}$ and then treated with $1 \% \mathrm{CuSO}_{4}$ for 5 min. After washing the section with deionized distilled $\mathrm{H}_{2} \mathrm{O}$, it was dehydrated in an ascending series of alcohols $(50 \%, 70 \%, 80 \%, 90 \%, 95 \%, 100 \% \times 2)$ and cleared in xylene. The section was mounted and then observed by microscopy. The use of DAB results in a brown insoluble compound at the site of cytochrome oxidase activity. In this experiment, we used COX-positive cells, which were stained brown when the cells have COX activity. Non-treatment control muscle was used as a positive control for COX staining where COX-positive cells were stained brown (Figure 5(a)). We also calculated the ratios of COX-positive cells and indicate the values on each image.

\section{Observations of mitochondrial membrane potentials in muscle tissue}

At $24 \mathrm{hr}$ postinjection, the rats were sacrificed and the crural muscles containing the gastrocnemius/soleus were harvested. The muscle tissues were treated with Tetramethylrhodamine (TMRM) and MitoTracker Deep Red 633 (MTDR) to stain mitochondria, and were then observed by confocal laser scanning microscopy (CLSM). TMRM, a conventional fluorescent stain for mitochondria, is easily washed out once the mitochondria experience a loss in membrane potential. On the other hand, MTDR is also selective for mitochondria and the stain is retained, even when mitochondrial membrane potential is lost. This experiment allowed us to evaluate the extent to which the hydrodynamic injection affected mitochondrial membrane potential. Briefly, the muscle tissues were incubated for 20-30 min with Hank's buffered salt solution containing TMRM (final concentration, $1 \mu \mathrm{M}$ ), MTDR (final concentration, $1 \mu \mathrm{M})$. Fluorescent images were obtained by CLSM (Nikon A1; Nikon Co. Ltd., Tokyo, Japan). The tissue specimens were excited with a wavelength of $561 \mathrm{~nm}$ from a DPSS laser. A series of images were obtained using a Nikon A1 confocal imaging system equipped with a water immersion objective lens (Plan Apo 60_1.20 PFS WI) and a 1st dichroic mirror (405/488/561/640). The two fluorescence detection channels (Ch) were set to the following filters: Ch1: 595/50 (red color) for TMRM and Ch2: 700/75 (cyan pseudo color) for MTDR. When the mitochondrial membrane potential was depolarized, the muscle 
tissues were treated with carbonyl cyanide 4-(trifluoromethoxy)phenylhydrazone (FCCP, Sigma), a mitochondrial uncoupler, before observation (final concentration of FCCP, $100 \mu \mathrm{M}$ ). 


\section{RESULTS}

\section{Gene delivery to skeletal muscle by HLV injection and evaluation of nuclear transgene expression}

We first evaluated nuclear transgene expression in hind limb muscle groups following HLV injection, to determine the optimal part of the limb muscle for the evaluation of mitochondrial gene delivery. In this experiment, pDNA encoding the luciferase gene was intravenously injected into the distal hind limb of rats, as shown in Fig. 1a.

Prior to each pDNA injection, a tourniquet was placed on the upper hind limb to restrict blood flow into and out of the hind limb. Basically, suspensions of naked pDNA (3 mL) containing $184 \mu \mathrm{g}$ pDNA were injected in $20 \mathrm{sec}$ into the dorsalis pedis vein at a distal site. After 2 min of injection, the tourniquet was released. At $24 \mathrm{hr}$ postinjection, the rats were sacrificed, limb muscles were harvested and were separated into three groups (quadriceps, hamstring, crural muscles). Nuclear transgene expression for each muscle group was then evaluated by measuring luciferase activity (Fig. 1b). a

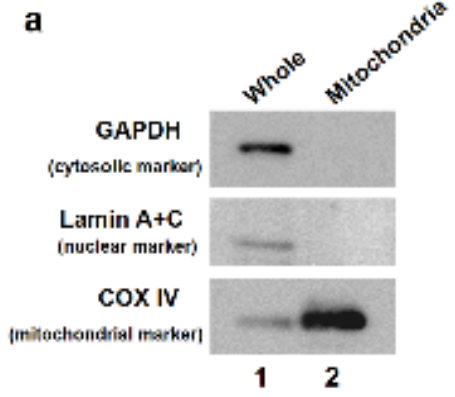

Fig. 2. Validation of mitochondrial delivery of pDNA targeted to skeletal muscle. (a) Western blot analysis of mitochondriaenriched fraction obtained from crural muscles. Each sample (3 $\mu \mathrm{g}$ of protein) was subjected to western blotting. Primary antibodies from mouse against the COX IV, Lamin $A+C$ and GAPDH, as mitochondrial, nuclear and cytosolic fraction marker, were used. Whole and Mitochondria indicate whole fraction containing homogenate of crural muscles and mitochondriaenriched fraction, respectively. (b) Evaluation of exogenous pDNA-levels in mitochondria-enriched fraction. Naked pDNA was injected into the distal of the limb by the HLV injection of naked. We also performed IM injection of naked pDNA at the quadriceps. The mitochondria-enriched fraction was isolated from the muscles at $24 \mathrm{hr}$ postinjection. PCR assays for D-loop and luciferase genes detection were performed in order to detect endogenous mtDNA and exogenous pDNA, respectively. Lane 1 , naked pDNA administration by HLV injection; lane 2, saline solution administration by HLV injection; lane 3, naked pDNA administration by IM injection.

The results showed that high luciferase activities were present and the activities were independent of the groups of limb muscles (quadriceps, hamstring and crural muscles, Fig. 1b), the mean values were higher than $10^{7} \mathrm{RLU} / \mathrm{mg}$ protein. Statistical analysis indicated that the values were comparable among Quadriceps, Hamstring and Crural muscles $(p=0.69)$. We also evaluated luciferase activities of the arm and liver, regions that are outside the hind limb, and no luciferase activity was detected (arm and liver, Fig. 1b). These results indicate that HLV injection from a distal site of the dorsalis pedis vein achieved effective nuclear gene transfer only in muscle groups of the lower limb distal to the tourniquet. We chose the crural muscle, where high and reproducible luciferase activity was observed, as a part of limb muscle for evaluating mitochondrial gene delivery. 


\section{Validation of mitochondrial delivery of pDNA targeted to skeletal muscle by HLV injection}

The evaluation of mitochondrial gene delivery involved the following procedures; HLV injection of pDNA into the distal limbs of rats (1), isolation of mitochondria from rat crural muscles (2), and detection/quantification of pDNA in the mitochondria-enriched fraction (3). To verify the mitochondrial delivery of pDNA, the purity of the isolated mitochondria needs to be verified. In this experiment, the crural muscles were harvested following HLV injection and homogenized, and a mitochondria-enriched fraction was then isolated from the homogenate by differential centrifugation, as described in a previous report $[27,28]$. Samples of the homogenate and mitochondria-enriched fraction were subjected to western blotting using primary antibodies against the COX IV, Lamin A+C and GAPDH, as mitochondrial, nuclear and cytosolic fraction markers (Fig. 2a). The data showed that only a band corresponding to COX IV was detected in the mitochondria-enriched fraction (lane 2 in Fig. 2a), indicating that mitochondria were exclusively isolated from the limb muscle.

We next attempted to detect pDNA in the mitochondria-enriched fraction using PCR at $24 \mathrm{hr}$ after an HLV injection of $184 \mu \mathrm{g}$ of pDNA (in $3 \mathrm{~mL}$ saline solution). Figure $2 \mathbf{b}$ shows agarose gel electrophoresis data for the PCR products derived from mtDNA and pDNA. In the case of the HLV injection of naked pDNA, a band corresponding to pDNA was observed (lanel in Fig. 2b), whereas an HLV injection of a saline solution showed no pDNA-band (lane2 in Fig. 2b). The results suggest that the presence of exogenous pDNA could be determined by PCR assays under these conditions, although the amounts of pDNA cannot be determined by this methodology. Moreover, we validated the mitochondrial delivery of pDNA following an IM injection (184 $\mu \mathrm{g}$ of pDNA in $100 \mu \mathrm{L}$ solution per one injection), as a control method. As a result, no pDNA band was observed in the case of the IM injection of naked pDNA (lane 3 in Fig. 2b). These results suggest that pDNA is delivered to myofibrillar mitochondria by HLV injection. 


\section{Effects of injection volume and dose on mitochondrial delivery by HLV injection of pDNA.}

To determine the a

optimal conditions for the mitochondrial delivery of pDNA by HLV injection, we investigated the effects of injection volume and dose on mitochondrial delivery by qPCR (Fig. 3). As a result, we observed the highest mitochondrial delivery when an injection volume of $3 \mathrm{~mL}$ containing $184 \mu \mathrm{g}$ of pDNA was administered $(\sim 1000 \mathrm{fg}$ $\mathrm{pDNA} / \mathrm{mg}$ muscle protein, b

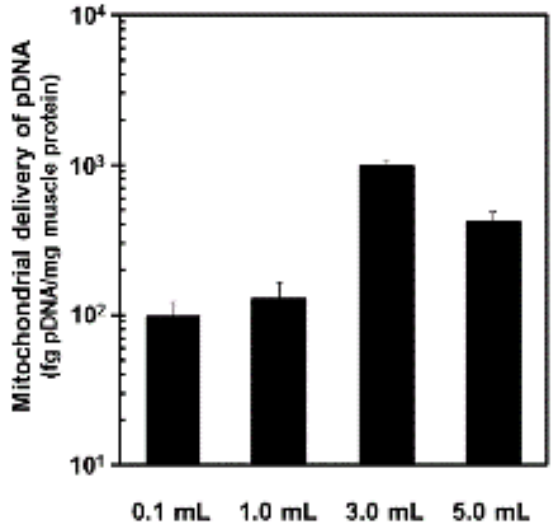

Applied volume [Applied dose of $184 \mu$ g]

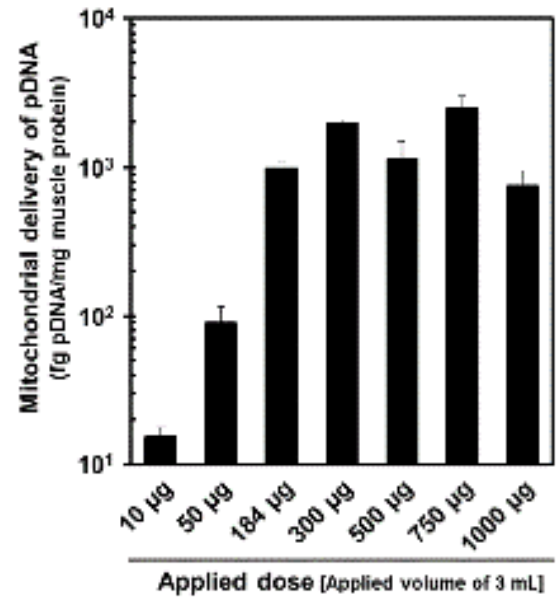

Applied dose [Applied volume of $3 \mathrm{~mL}]$
Fig. 3. Effects of injection volume (a) and dose of pDNA (b) on mitochondrial delivery by HLV injection of pDNA. At $24 \mathrm{hr}$ after HLV injection under various condition, the crural muscles were harvested, and the pDNA in mitochondria-enriched fraction was then measured using q-PCR. Bars indicate means \pm S.E. $(n=3-7)$.

Fig. 3a), while the value was less than 20 when a saline solution was injected under similar condition.

We also confirmed that the mitochondrial delivery was dose dependent (when $3 \mathrm{~mL}$ of injection volume), and that higher levels of $\sim 1000 \mathrm{fg} \mathrm{pDNA} / \mathrm{mg}$ muscle protein were achieved when more than $184 \mu \mathrm{g}$ of pDNA was injected (Fig. 3b). Based on these results, we concluded that the injection of $184 \mu \mathrm{g}$ of pDNA in $3 \mathrm{~mL}$ of solution was the optimal condition for the mitochondrial delivery when HLV injection was performed in the case of naked pDNA, and hereafter we used this protocol.

We next confirmed that the HLV injection technique was sufficient for delivering exogenous pDNA inside a mitochondrion, based on the DNase I digestion experiment (Fig. 4). As a result, the levels of exogenous pDNA in the mitochondria-enriched fraction following the HLV injection of pDNA (184 $\mu \mathrm{g}, 3 \mathrm{~mL}$ ) were comparable before and after the DNase I treatment (Fig. 4), indicating that no pDNA was detected outside mitochondria in the mitochondria-enriched fraction. Regarding this result, the procedure used to obtain the mitochondria-enriched fraction might remove pDNA

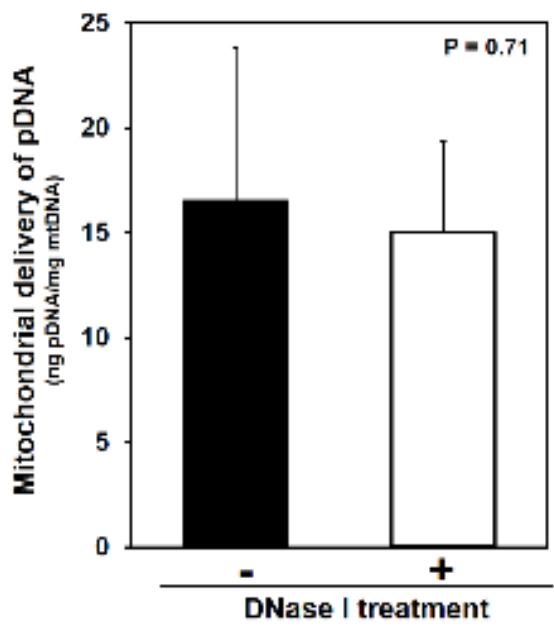

Fig. 4. Detection of exogenous pDNA in the mitochondria-enriched fraction before and after DNase treatment. At $24 \mathrm{hr}$ after HLV injection, the crural muscles were harvested, and the pDNA in mitochondria-enriched fraction was then measured using q-PCR, before and after treatment with DNase I. Bars indicate means \pm S.D. $(n=3)$. Statistical analysis was performed by a two-tailed unpaired Student's t-test $(p=0.71)$. 
from the mitochondrial surface, probably because anionic pDNA is unable to bind tightly to mitochondria with a high negative charge.

To visualize the delivery of pDNA to skeletal muscle following the HLV injection, Immuno-FISH analysis was performed on frozen tissue sections (Figure S1). The results showed that signals corresponding to exogenous pDNA were present in the soleus muscle, and that the pDNA was nonspecifically distributed. We also observed several yellow signals, suggesting that several pDNA molecules might have been nonspecifically localized on myofibrillar mitochondria. 


\section{Evaluation of mitochondrial toxicity in skeletal muscle after HLV injection}

\author{
Myofibrillar \\ mitochondrial activity was \\ evaluated by COX staining, \\ after performing an HLV \\ injection. As shown in
} Figure 5, non-treatment control muscle (a), saline administered muscle (b) and naked pDNA administered muscle (c) were observed. Non-treatment control muscle was used as a positive control of $\mathrm{COX}$
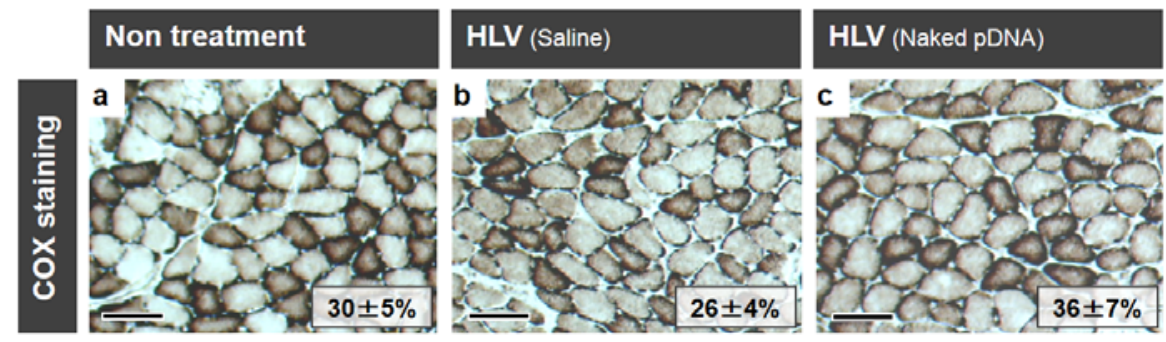

Fig. 5. COX staining on skeletal muscle following HLV injection. Frozen cross-sections (10 $\mu \mathrm{m}$ thickness) of HLV injection performed skeletal muscle were prepared, followed by COX staining. The section was then observed by microscopy; non-treatment control muscle (a), saline administered muscle (b) and naked pDNA administered muscle (c). Scale bars, $100 \mu \mathrm{m}$. In this experiment, cells were stained brown when the cells have the cytochrome oxidase activity (COX-positive cells). Non-treatment control muscle was used as positive control where COX-positive cells were stained brown (a).We also calculated the ratios of COX-positive cells and indicate the values on each image. Data represent means \pm S.D. $(n=3-4)$. Statistical analysis was performed by one-way ANOVA $(p=0.10)$.

staining where COX-positive cells were stained brown (Figure 5(a)).We also calculated the ratios of COX-positive cells and performed by one-way ANOVA. As a result, we found no significant differences between non-treatment control muscles and others $(p=0.10)$, indicating that the mitochondria maintained COX activity in skeletal muscles after the HLV injection.

Mitochondrial membrane potentials of the soleus of crural muscle following HLV injection were evaluated (Fig. 6). In this experiment, the mitochondria in soleus muscle were stained with TMRM (red color; a, c, e, g, Fig. 6)

and MTDR (cyan pseudo color; b, d, f, h, Fig. 6). The staining of mitochondria with TMRM is dependent on the membrane potential, while MTDR stains mitochondria, even when the membrane potential is lost. In the case of saline administered
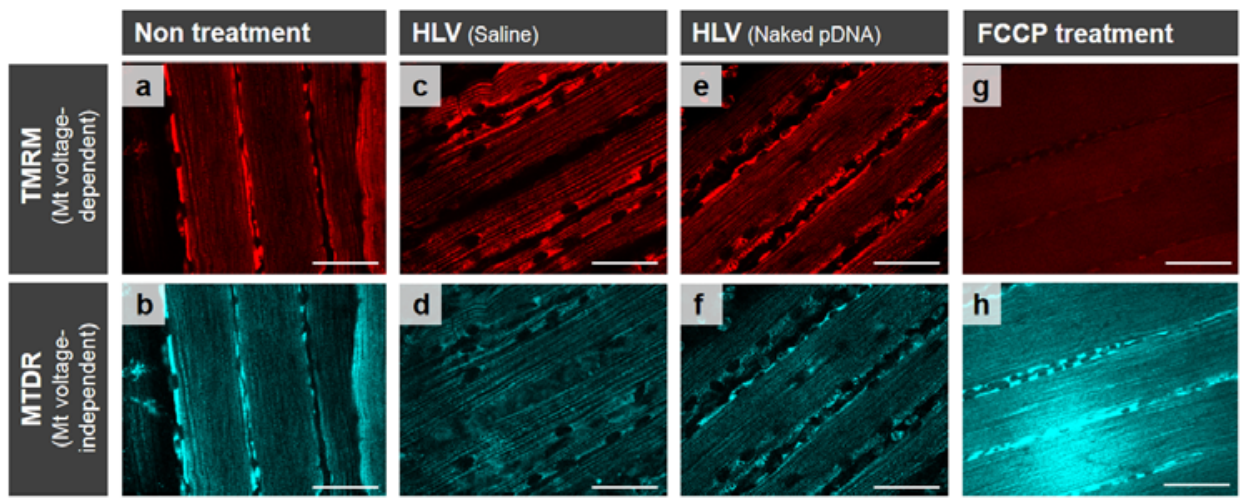

Fig. 6. Observation of skeletal muscle after staining mitochondria following HLV injection. At $24 \mathrm{hr}$ post HLV injections, the soleus of crural muscles were harvested, and mitochondria were then stained with Tetramethylrhodamine (TMRM) (red color; a, c, e, g) and MitoTracker Deep Red 633 (MTDR) (cyan pseudo color; b, d, f, h). The staining of mitochondria with TMRM is dependent on the membrane potential, while MTDR can stain mitochondria even when membrane potential is lost. The muscle tissues were observed using CLSM. Scale bars, $50 \mu \mathrm{m}$. 
muscle (c, d), naked pDNA administered muscle (e, f), the mitochondria were extensively stained with TMRM at a level comparable to that for non-treatment muscle (a, b) (Fig. 6), indicating that most of the mitochondria in skeletal muscles maintained their membrane potential. We also confirmed that TMRM stained mitochondria incompletely, when the mitochondrial membrane potential was depolarized, in the case where muscles were treated with FCCP (a mitochondrial uncoupler) ( $g$, $h$ in Fig. 6). These results indicate that the HLV injection of pDNA does not significantly decrease the mitochondrial membrane potential, compared to FCCP treatment. A similar tendency regarding mitochondrial membrane potentials was observed in the case of the gastrocnemius of crural muscles (Fig S2).

\section{DISCUSSION}

To date, several groups have reported successful examples of mitochondrial RNA delivery [2931], since the fact that the mitochondrion possesses endogenous RNA imported via an RNA signal tag system in these studies [32,33]. Moreover, it has been reported that allotropic expression in the nucleus, followed by the transfer of a signal tag fused protein to the mitochondria appears promising [11]. However, these signal tags may not function in mitochondrial diseased cells. For example, the signal tags could not be used to correct defects in the mitochondrial protein import [34] of their cargo. Similarly, signal tags may be of little benefit in the case of mtDNA-encoded-proteins, as these proteins are generally too hydrophobic to maintain the unfolded conformation that is required for signal tag-mediated import [35]. In this study, we show the possibility that HLV injection is capable of achieving mitochondrial gene delivery targeted to skeletal muscle tissue of mammals. This methodology could be a novel gene delivery system directly targeting mitochondria by hydrodynamic force.

Hydrodynamic-based gene delivery has been defined as the rapid injection of a high-volume of naked pDNA solution into blood vessels [21, 22]. This technique was first reported by Liu's group and Wollf's group in 1999, where effective nuclear transgene expression in hepatocytes in mice was achieved by hydrodynamic tail vein injection. In 2004, Wolff's group showed that the rapid injection of large volumes of naked pDNA into the great saphenous vein of the distal hind limb of a rat (HLV injection) resulted in effective nuclear transgene expression in skeletal muscle [20]. Based on previous great reports, we attempted to achieve mitochondrial delivery of pDNA targeted to skeletal muscle using HLV injection. We evaluated nuclear transgene expression following previous protocol of HLV injection (injection volume, $3 \mathrm{~mL}$; injection rate, approximately $10 \mathrm{~mL} / \mathrm{min}$ ), although the injection site was different, the dorsalis pedis vein. As shown in Figure 1, a high nuclear transgene expression was observed in all groups of limb muscles, especially the values for the hamstring and crural muscles were high and reproducible. 
Our results are consistent with previous reports by Wolff's group where effective nuclear transgene was observed in muscle near the injection site [20].

We confirmed that the conditions for the DNase I treatment are adequate for the detection of exogenous pDNA in the mitochondria-enriched fraction following HLV injection (Fig. S3). In this experiment, the mitochondria-enriched fraction was mixed with pDNA, and the pDNA was then quantified by q-PCR before and after DNase I digestion (see Supplementary Methods for details). The results showed that a large amount of pDNA that was mixed with the mitochondria-enriched fraction was digested by DNase I under these conditions (Fig. S3a). We also observed that the value was 55 before DNase I treatment, when $1 \mu \mathrm{g}$ of pDNA was mixed with the mitochondria-enriched fraction in this condition (closed column in Fig. S3b). Although this value is 3-fold higher than that for the HLV injection (value = 15, Fig. 4), it decreased to 1.6 after DNase I treatment (open column in Fig. S3b). On the other hand, the values were comparable before and after DNase I treatment, when HLV injection was performed (Fig. 4). These results support the conclusion that this DNase I treatment is sufficient for digesting pDNA in the mitochondria-enriched fraction following HLV injection, when the pDNAs are present outside the mitochondrion.

Our results suggest that HLV injection delivered pDNA into mitochondria, although the mechanism is unknown. Here, we discuss how pDNA overcome the mitochondrial membrane. Previously, it has been reported that isolated mitochondria can import linear DNA in plant [36], mammalian [37] and Saccharomyces cerevisiae [38]. Moreover, Ibrahim et al showed that linear turnip $11.6 \mathrm{~kb}$ mitochondrial pDNA was efficiently internalized into human mitochondria in a sequence-independent manner [39]. These previous reports can support our result that cytosolic pDNA that is internalized by hydrodynamic force was delivered into mitochondria. On the other hand, Kolintchenko et al reported that circular pDNA was not delivered into mitochondria in the plant [36]. Thus, we also presumed other mechanisms of mitochondrial delivery of circular pDNA in mammalian. One possibility is that hydrodynamic force might induce the transient opening of mitochondrial membrane, similar to the case of cellular membrane. An alternative possibility is that mitochondrial uptake of circular pDNA occurs during mitochondrial fusion and fission, since mitochondria in living cells form a continuous, highly dynamic, reticulum or network, that is continually undergoing fusion and fission [40,41]. This important issue are required to investigate in the future, although the details of the mechanism responsible for this are currently unclear.

Since the efficient delivery of pDNA using HLV injection requires severe conditions (large injection volume, $3 \mathrm{~mL}$; high injection rate, $10 \mathrm{~mL} / \mathrm{min}$ ), an assessment of the toxicity is an important issue that relates to the potential therapeutic utility of this methodology. To date, Wolff's group performed a detailed assessment of toxicities following HLV injection, and indicated minimal and transient muscle damage on the basis of histological analyses, creatine kinase measurements, and $\mathrm{T}_{2}$-weighted magnetic 
resonance imaging scans $[42,43]$. In this study, we investigated mitochondrial toxicity following HLV injection, because such information is not available. The evaluation of COX activity (Fig 5) and mitochondrial membrane potentials (Fig 6) showed that HLV injection did not significantly affect mitochondrial function. 


\section{CONCLUSION}

In this study, we attempted the mitochondrial delivery of exogenous pDNA in skeletal muscle using HLV injection. Our results indicated that an HLV injection resulted in the mitochondrial delivery of naked pDNA. Moreover, the evaluation of COX activity and mitochondrial membrane potentials showed that HLV injection was not toxic to mitochondria. The findings are the first report regarding in vivo mitochondrial gene delivery using the HLV injection procedure. We expected that the findings reported herein will contribute to the development of a mitochondrial gene delivery system, resulting in the achievement of mitochondrial gene diagnosis and therapy and will accelerate research regarding mitochondrial molecular biology. Future studies will involve attempts to achieve in vivo mitochondrial transgene expression by HLV injection in conjunction with experts in mitochondrial molecular biology. Studies directed toward this goal are currently in progress.

\section{ACKNOWLEDGMENT}

This work was supported, in part by, the Advanced research for medical products Mining Programme of the National Institute of Biomedical Innovation (NIBIO), a Grant-in-Aid for Young Scientists (A) and a Grant-in-Aid for Scientific Research (S) from the Ministry of Education, Culture, Sports, Science and Technology, the Japanese Government (MEXT). We thank Sapporo General Pathology Laboratory Co., Ltd., for the technical assistance with the histopathology (Sapporo, Japan). We are grateful to Dr. Dexi Liu for his critical reading of the manuscript and helpful comments. We thank Chromosome Science Labo Inc. (Sapporo, Japan) for FISH analysis and Sapporo General Pathology Laboratory Co., Ltd. (Sapporo, Japan) for the technical assistance with the histopathology. We also thank Dr. Milton Feather for his helpful advice in writing the manuscript. 


\section{FIGURE LEGENDS.}

Figure 1. Schematic images of gene delivery to skeletal muscle by HLV injection and evaluation of nuclear transgene expression in part of skeletal muscles. (a) Schematic diagram of HLV injection of pDNA into distal hind limb of rats. pDNA was intravenously injected into the dorsalis pedis vein under the conditions that restrict blood flow with a tourniquet. At $24 \mathrm{hr}$ postinjection, rats were sacrificed, the limb muscles were harvested and separated into three groups (quadriceps, hamstring, crural muscles). (b) Gene expression in part of skeletal muscles. Luciferase activities were measured at $24 \mathrm{hr}$ after HLV injection of pDNA coding luciferase. Bars represent the mean values $(n=4)$. Statistical analysis among Quadriceps, Hamstring and Crural muscles was performed by a one-way ANOVA ( $p=0.69)$. We also measured luciferase activities in the arm and liver as controls. N.D., not determined.

Figure 2. Validation of mitochondrial delivery of pDNA targeted to skeletal muscle. (a) Western blot analysis of mitochondria-enriched fraction obtained from crural muscles. Each sample ( $3 \mu \mathrm{g}$ of protein) was subjected to western blotting. Primary antibodies from mouse against the COX IV, Lamin A+C and GAPDH, as mitochondrial, nuclear and cytosolic fraction marker, were used. Whole and Mitochondria indicate whole fraction containing homogenate of crural muscles and mitochondria-enriched fraction, respectively. (b) Evaluation of exogenous pDNA-levels in mitochondria-enriched fraction. Naked pDNA was injected into the distal of the limb by the HLV injection of naked. We also performed the IM injection of naked pDNA at the quadriceps. The mitochondria-enriched fraction was isolated from the muscles at $24 \mathrm{hr}$ postinjection. PCR assays for D-loop and luciferase genes detection were performed in order to detect endogenous mtDNA and exogenous pDNA, respectively. Lane 1, naked pDNA administration by HLV injection; lane 2, saline solution administration by HLV injection; lane 3, naked pDNA administration by IM injection.

Figure 3. Effects of injection volume (a) and dose of pDNA (b) on mitochondrial delivery by HLV injection of pDNA. At $24 \mathrm{hr}$ after HLV injection under various condition, the crural muscles were harvested, and the pDNA in mitochondria-enriched fraction was then measured using q-PCR. Bars indicate means \pm S.E. $(n=3-7)$.

Figure 4. Detection of exogenous pDNA in the mitochondria-enriched fraction before and after DNase treatment. At $24 \mathrm{hr}$ after HLV injection, the crural muscles were harvested, and the pDNA in mitochondria-enriched fraction was then measured using q-PCR, before and after treatment with DNase I. Bars indicate means \pm S.D. $(n=3)$. Statistical analysis was performed by a two-tailed unpaired Student's t-test $(\mathrm{p}=0.71)$. 
Figure 5. COX staining on skeletal muscle following HLV injection. Frozen cross-sections $(10 \mu \mathrm{m}$ thickness) of HLV injection performed skeletal muscle were prepared, followed by COX staining. The section was then observed by microscopy; non-treatment control muscle (a), saline administered muscle (b) and naked pDNA administered muscle (c). Scale bars, $100 \mu \mathrm{m}$. In this experiment, cells were stained brown when the cells have the cytochrome oxidase activity (COX-positive cells). We also calculated the ratios of COX-positive cells and indicate the values on each image. Data represent means \pm S.D. (n= 3 4). Statistical analysis was performed by one-way ANOVA $(p=0.10)$.

Figure 6. Observation of skeletal muscle after staining mitochondria following HLV injection. At $24 \mathrm{hr}$ post HLV injections, the soleus of crural muscles were harvested, and mitochondria were then stained with TMRM (red color; a, c, e, g) and MTDR (cyan pseudo color; b, d, f, h). The staining of mitochondria with TMRM is dependent on the membrane potential, while MTDR can stain mitochondria even when membrane potential is lost. The muscle tissues were observed using CLSM. Scale bars, $50 \mu \mathrm{m}$. 


\section{REFERENCES.}

[1] D.C. Chan, Mitochondria: dynamic organelles in disease, aging, and development, Cell, 125 (2006) 1241-1252.

[2] I.J. Holt, A.E. Harding, J.A. Morgan-Hughes, Deletions of muscle mitochondrial DNA in patients with mitochondrial myopathies, Nature, 331 (1988) 717-719.

[3] A.H. Schapira, Mitochondrial diseases, Lancet, 379 (2012) 1825-1834.

[4] D.C. Wallace, Mitochondrial diseases in man and mouse, Science, 283 (1999) 1482-1488.

[5] Y. Goto, I. Nonaka, S. Horai, A mutation in the tRNA(Leu)(UUR) gene associated with the MELAS subgroup of mitochondrial encephalomyopathies, Nature, 348 (1990) 651-653.

[6] S. Shanske, C.T. Moraes, A. Lombes, A.F. Miranda, E. Bonilla, P. Lewis, M.A. Whelan, C.A. Ellsworth, S. DiMauro, Widespread tissue distribution of mitochondrial DNA deletions in Kearns-Sayre syndrome, Neurology, 40 (1990) 24-28.

[7] J.M. Shoffner, M.T. Lott, A.M. Lezza, P. Seibel, S.W. Ballinger, D.C. Wallace, Myoclonic epilepsy and ragged-red fiber disease (MERRF) is associated with a mitochondrial DNA tRNA(Lys) mutation, Cell, 61 (1990) 931-937.

[8] V. Weissig, From serendipity to mitochondria-targeted nanocarriers, Pharm Res, 28 (2011) 2657-2668.

[9] Y. Yamada, H. Akita, H. Harashima, Multifunctional envelope-type nano device (MEND) for organelle targeting via a stepwise membrane fusion process, Methods Enzymol, 509 (2012) 301-326.

[10] S. Biswas, N.S. Dodwadkar, P.P. Deshpande, V.P. Torchilin, Liposomes loaded with paclitaxel and modified with novel triphenylphosphonium-PEG-PE conjugate possess low toxicity, target mitochondria and demonstrate enhanced antitumor effects in vitro and in vivo, J Control Release, 159 (2012) 393-402. [11] Y.W. Won, K.S. Lim, Y.H. Kim, Intracellular organelle-targeted non-viral gene delivery systems, J Control Release, 152 (2011) 99-109.

[12] Y. Zhang, A. Satterlee, L. Huang, In Vivo Gene Delivery by Nonviral Vectors: Overcoming Hurdles?, Mol Ther, (2012).

[13] K. Itaka, K. Kataoka, Recent development of nonviral gene delivery systems with virus-like structures and mechanisms, Eur J Pharm Biopharm, 71 (2009) 475-483.

[14] K. Kogure, H. Akita, Y. Yamada, H. Harashima, Multifunctional envelope-type nano device (MEND) as a non-viral gene delivery system, Adv Drug Deliv Rev, 60 (2008) 559-571.

[15] $\mathrm{H}$. Yu, E. Wagner, Bioresponsive polymers for nonviral gene delivery, Curr Opin Mol Ther, 11 (2009) 165-178.

[16] H. Akita, K. Kogure, R. Moriguchi, Y. Nakamura, T. Higashi, T. Nakamura, S. Serada, M. Fujimoto, T. Naka, S. Futaki, H. Harashima, Reprint of: Nanoparticles for ex vivo siRNA delivery to dendritic cells for cancer vaccines: Programmed endosomal escape and dissociation, J Control Release, 149 (2011) 5864.

[17] C.I. Wooddell, J.O. Hegge, G. Zhang, M.G. Sebestyen, M. Noble, J.B. Griffin, L.V. Pfannes, H. Herweijer, J.E. Hagstrom, S. Braun, T. Huss, J.A. Wolff, Dose response in rodents and nonhuman primates after hydrodynamic limb vein delivery of naked plasmid DNA, Hum Gene Ther, 22 (2011) 889903.

[18] J.O. Hegge, C.I. Wooddell, G. Zhang, J.E. Hagstrom, S. Braun, T. Huss, M.G. Sebestyen, M.E. Emborg, J.A. Wolff, Evaluation of hydrodynamic limb vein injections in nonhuman primates, Hum Gene Ther, 21 (2010) 829-842.

[19] H. Herweijer, J.A. Wolff, Gene therapy progress and prospects: hydrodynamic gene delivery, Gene Ther, 14 (2007) 99-107.

[20] J.E. Hagstrom, J. Hegge, G. Zhang, M. Noble, V. Budker, D.L. Lewis, H. Herweijer, J.A. Wolff, A facile nonviral method for delivering genes and siRNAs to skeletal muscle of mammalian limbs, Mol Ther, 10 (2004) 386-398. 
[21] F. Liu, Y. Song, D. Liu, Hydrodynamics-based transfection in animals by systemic administration of plasmid DNA, Gene Ther, 6 (1999) 1258-1266.

[22] G. Zhang, V. Budker, J.A. Wolff, High levels of foreign gene expression in hepatocytes after tail vein injections of naked plasmid DNA, Hum Gene Ther, 10 (1999) 1735-1737.

[23] S. Yan, Q. Fu, Y. Zhou, J. Wang, Y. Liu, X. Duan, S. Jia, J. Peng, B. Gao, J. Du, Q. Zhou, Y. Li, X. Wang, L. Zhan, High levels of gene expression in the hepatocytes of adult mice, neonatal mice and tree shrews via retro-orbital sinus hydrodynamic injections of naked plasmid DNA, J Control Release, 161 (2012) 763-771.

[24] K. Itaka, K. Osada, K. Morii, P. Kim, S.H. Yun, K. Kataoka, Polyplex nanomicelle promotes hydrodynamic gene introduction to skeletal muscle, J Control Release, 143 (2010) 112-119.

[25] K. Osada, T. Shiotani, T.A. Tockary, D. Kobayashi, H. Oshima, S. Ikeda, R.J. Christie, K. Itaka, K. Kataoka, Enhanced gene expression promoted by the quantized folding of pDNA within polyplex micelles, Biomaterials, 33 (2012) 325-332.

[26] S. Uchida, K. Itaka, Q. Chen, K. Osada, K. Miyata, T. Ishii, M. Harada-Shiba, K. Kataoka, Combination of chondroitin sulfate and polyplex micelles from Poly(ethylene glycol)-poly\{N'-[N-(2aminoethyl)-2-aminoethyl]aspartamide block copolymer for prolonged in vivo gene transfection with reduced toxicity, J Control Release, 155 (2011) 296-302.

[27] Y. Shinohara, M.R. Almofti, T. Yamamoto, T. Ishida, F. Kita, H. Kanzaki, M. Ohnishi, K. Yamashita, S. Shimizu, H. Terada, Permeability transition-independent release of mitochondrial cytochrome c induced by valinomycin, Eur J Biochem, 269 (2002) 5224-5230.

[28] Y. Yamada, H. Akita, H. Kamiya, K. Kogure, T. Yamamoto, Y. Shinohara, K. Yamashita, H. Kobayashi, H. Kikuchi, H. Harashima, MITO-Porter: A liposome-based carrier system for delivery of macromolecules into mitochondria via membrane fusion, Biochimica et biophysica acta, 1778 (2008) 423-432.

[29] B. Mahata, S. Mukherjee, S. Mishra, A. Bandyopadhyay, S. Adhya, Functional delivery of a cytosolic tRNA into mutant mitochondria of human cells, Science (New York, N.Y, 314 (2006) 471-474.

[30] G. Wang, E. Shimada, J. Zhang, J.S. Hong, G.M. Smith, M.A. Teitell, C.M. Koehler, Correcting human mitochondrial mutations with targeted RNA import, Proc Natl Acad Sci U S A, 109 (2012) 48404845.

[31] O.A. Kolesnikova, N.S. Entelis, C. Jacquin-Becker, F. Goltzene, Z.M. Chrzanowska-Lightowlers, R.N. Lightowlers, R.P. Martin, I. Tarassov, Nuclear DNA-encoded tRNAs targeted into mitochondria can rescue a mitochondrial DNA mutation associated with the MERRF syndrome in cultured human cells, Hum Mol Genet, 13 (2004) 2519-2534.

[32] T. Endo, K. Yamano, T. Yoshihisa, Mitochondrial matrix reloaded with RNA, Cell, 142 (2010) 362363.

[33] G. Wang, H.W. Chen, Y. Oktay, J. Zhang, E.L. Allen, G.M. Smith, K.C. Fan, J.S. Hong, S.W. French, J.M. McCaffery, R.N. Lightowlers, H.C. Morse, 3rd, C.M. Koehler, M.A. Teitell, PNPASE regulates RNA import into mitochondria, Cell, 142 (2010) 456-467.

[34] L. Tranebjaerg, C. Schwartz, H. Eriksen, S. Andreasson, V. Ponjavic, A. Dahl, R.E. Stevenson, M. May, F. Arena, D. Barker, et al., A new X linked recessive deafness syndrome with blindness, dystonia, fractures, and mental deficiency is linked to Xq22, Journal of medical genetics, 32 (1995) 257-263.

[35] R.I. Owen, A.P. Lewin, A. Peel, J. Wang, J. Guy, W.W. Hauswirth, P.W. Stacpoole, T.R. Flotte, Recombinant adeno-associated virus vector-based gene transfer for defects in oxidative metabolism, Hum Gene Ther, 11 (2000) 2067-2078.

[36] M. Koulintchenko, Y. Konstantinov, A. Dietrich, Plant mitochondria actively import DNA via the permeability transition pore complex, The EMBO journal, 22 (2003) 1245-1254.

[37] M. Koulintchenko, R.J. Temperley, P.A. Mason, A. Dietrich, R.N. Lightowlers, Natural competence of mammalian mitochondria allows the molecular investigation of mitochondrial gene expression, Hum Mol Genet, 15 (2006) 143-154. 
[38] F. Weber-Lotfi, N. Ibrahim, P. Boesch, A. Cosset, Y. Konstantinov, R.N. Lightowlers, A. Dietrich, Developing a genetic approach to investigate the mechanism of mitochondrial competence for DNA import, Biochim Biophys Acta, 1787 (2009) 320-327.

[39] N. Ibrahim, H. Handa, A. Cosset, M. Koulintchenko, Y. Konstantinov, R.N. Lightowlers, A. Dietrich, F. Weber-Lotfi, DNA delivery to mitochondria: sequence specificity and energy enhancement, Pharm Res, 28 (2011) 2871-2882.

[40] J. Bereiter-Hahn, M. Voth, Dynamics of mitochondria in living cells: shape changes, dislocations, fusion, and fission of mitochondria, Microscopy research and technique, 27 (1994) 198-219.

[41] B. Westermann, Mitochondrial fusion and fission in cell life and death, Nature reviews. Molecular cell biology, 11 (2010) 872-884.

[42] H. Toumi, J. Hegge, V. Subbotin, M. Noble, H. Herweijer, T.M. Best, J.E. Hagstrom, Rapid intravascular injection into limb skeletal muscle: a damage assessment study, Mol Ther, 13 (2006) 229236.

[43] K.K. Vigen, J.O. Hegge, G. Zhang, R. Mukherjee, S. Braun, T.M. Grist, J.A. Wolff, Magnetic resonance imaging-monitored plasmid DNA delivery in primate limb muscle, Hum Gene Ther, 18 (2007) 257-268. 


\section{SUPPLEMENTARY MATERIAL}

\section{Supplementary Methods}

\section{Visualization of pDNA delivered to muscle tissue by Immuno-FISH analysis}

At $24 \mathrm{hr}$ postinjection, the rats were sacrificed and the soleus of the crural muscles harvested, Immuno-FISH was performed on frozen tissue sections. In a typical run, $5-\mu \mathrm{m}$ thick sections were washed in phosphate-buffered saline [PBS (-)] for 5 min, fixed with 4\% paraformaldehyde / PBS (-) at $4^{\circ} \mathrm{C}$ for 7 min, digested in pepsin solution $(0.02 \%$ in $0.1 \mathrm{~N} \mathrm{HCl})$ at $37^{\circ} \mathrm{C}$ for $4 \mathrm{~min}$, and dehydrated. pDNA were labeled by nick translation with digoxigenin-dUTP (Roche Diagnostics; Mannheim, Germany). Probes were applied to the pretreated sections, covered with cover slips and simultaneously denatured at $80^{\circ} \mathrm{C}$ for 10 min. Hybridization was carried out at $37^{\circ} \mathrm{C}$ overnight. Sections were then washed with $50 \%$ formamide $/ 2 \mathrm{xSSC}$ at $37^{\circ} \mathrm{C}$ for $20 \mathrm{~min}, 1 \mathrm{xSSC}$ for $15 \mathrm{~min}$ at RT. For additional immunofluorescent staining with FISH, the mouse anti-COX IV were diluted to $1: 500$ concentrations with 5\% skim milk, $0.1 \%$ nonidet $\mathrm{P}-40$ in PBS and applied to the FISH samples at $37^{\circ} \mathrm{C}$ for $1 \mathrm{hr}$. To detect FISH signals and immunofluorescent signal, the second antibodies (anti-mouse Alexa488 [1:500], anti-digoxigenine Cy5 [1:500]) were applied to the samples at $37^{\circ} \mathrm{C}$ for $30 \mathrm{~min}$. Nuclei were visualized with DAPI. Samples were examined by fluorescent microscopy (Leica CW-4000 System, Leica Microsystems, Wetzlar, Germany) and Leica software.

\section{DNase treatment of mitochondria-enriched fraction mixed with various amount of pDNA}

Rats were sacrificed and the crural muscles were harvested, and a mitochondria-enriched fraction was then obtained from the tissue, as described in the main text. The mitochondria-enriched fraction was mixed with various amounts of pDNA, and DNase digestion was then performed. We next extracted the pDNA and mtDNA from the resulting suspension, and the amount of pDNA and mtDNA were measured by quantitative PCR to determine the value of pDNA (ng pDNA/ mg mtDNA). DNase digestion, DNA extraction and quantitative PCR were performed, as described above. 


\section{Supplementary Results}

1. Visualization of pDNA in skeletal muscle following HLV injection by Immuno-FISH analysis.

To visualize the delivery of pDNA in skeletal muscle following the HLV injection, rats were sacrificed at $24 \mathrm{hr}$ postinjection, the soleus of the crural muscle was harvested, and an Immuno-FISH analysis was then performed on frozen tissue sections. In this experiment, we immunostained the soleus of the crural muscle with COX IV, a mitochondrial protein (pseudo red color in Fig. S1a) followed by FISH to detect pDNA (pseudo green color in Fig. S1b). As a result, we succeeded in detecting signals of exogenous pDNA in the soleus muscle, the pDNA was nonspecifically distributed. We also observed several yellow signals, suggesting that several pDNA molecules might be localized on myofibrillar mitochondria nonspecifically (Fig. S1c). 
Figure S1. Visualization of pDNA in myofibrillar mitochondria by Immuno-FISH analysis.

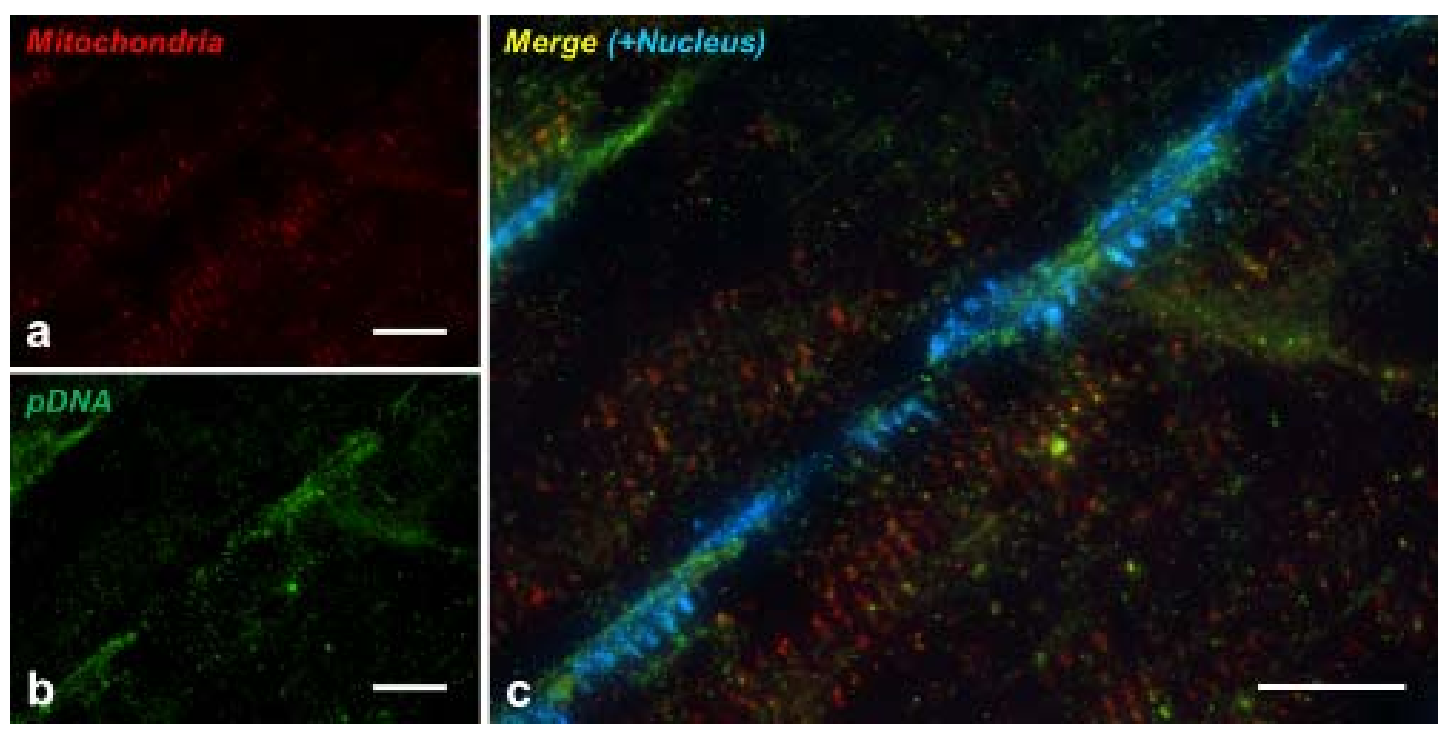

Frozen longitudinal-sections (10 $\mu \mathrm{m}$-thickness) of soleus of crural muscle were prepared after HLV injection of pDNA, and the sections were immunostained with COX IV, a mitochondrial protein (a, pseudo red color) followed by FISH to detect pDNA (b, pseudo green color). Nuclei were stained with DAPI (blue). The clusters present in the mitochondria (yellow) are indicated in merge image (c). Scale bars, $10 \mu \mathrm{m}$. 
2. Evaluation of mitochondrial membrane potentials in gastrocnemius of crural muscles following HLV injection.

We observed mitochondria in the gastrocnemius of crural muscles stained with TMRM (red color) and MTDR (cyan pseudo color) to evaluate the mitochondrial membrane potential after HLV injection (Fig. S2). The staining of mitochondria with TMRM is dependent on the membrane potential, while MTDR stains mitochondria, even when the membrane potential is lost. In the case of saline administered muscle $(\mathrm{c}, \mathrm{d})$, naked pDNA administered muscle (e, f), the mitochondria were extensively stained with TMRM at a level comparable to that for non-treated muscle $(a, b)$ (Fig. S2), indicating that most of the mitochondria in skeletal muscles maintained their membrane potential. 
Figure S2. Observation of gastrocnemius muscle after staining mitochondria with TMRM following HLV injection.

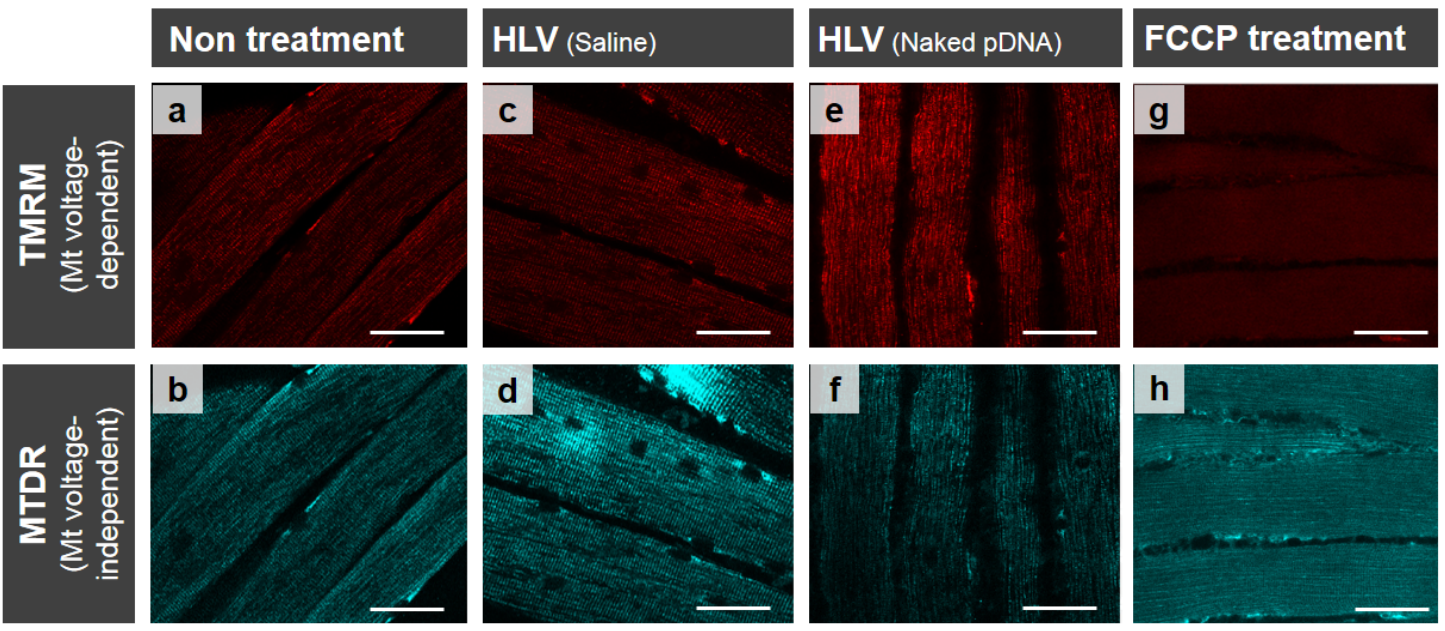

At $24 \mathrm{hr}$ after the HLV injections, the gastrocnemius of crural muscles were harvested, and the mitochondria were then stained with Tetramethylrhodamine (TMRM) (red color; a, c, e, g) and MitoTracker Deep Red 633 (MTDR) (cyan pseudo color; b, d, f, h). The muscle tissues were observed by CLSM. FCCP, carbonyl cyanide 4-(trifluoromethoxy)phenylhydrazone (a mitochondrial uncoupler). Scale bars, $50 \mu \mathrm{m}$. 
3. Validation of DNase treatment for the detection of exogenous pDNA in the mitochondria-enriched fraction

We confirmed that the conditions used for the DNase I treatment are adequate for the detection of exogenous pDNA in the mitochondria-enriched fraction following HLV injection. In this experiment, the mitochondria-enriched fraction was mixed with pDNA, and the value of pDNA was then quantified by quantitative PCR before and after DNase digestion as described in Supplementary Methods. The results showed that a large amount of pDNA mixed with the mitochondria-enriched fraction was digested by DNase I under this condition (Fig. S3a).

We also observed that the value was 55 before DNase I treatment, when $1 \mu \mathrm{g}$ of pDNA was mixed with the mitochondria-enriched fraction under these conditions (closed column in Fig. S3b). Although this value is 3-fold higher than that for the HLV injection of pDNA (value =15, Fig. 4), it decreased to 1.6 after the DNase I treatment (open column in Fig. S3b). These results support the conclusion that this DNase I treatment is sufficient for to digest the naked pDNA in the mitochondria-enriched fraction following HLV injection, when the pDNAs are present outside the mitochondria. 
Figure S3. DNase treatment of mitochondria-enriched fraction mixed with of pDNA.
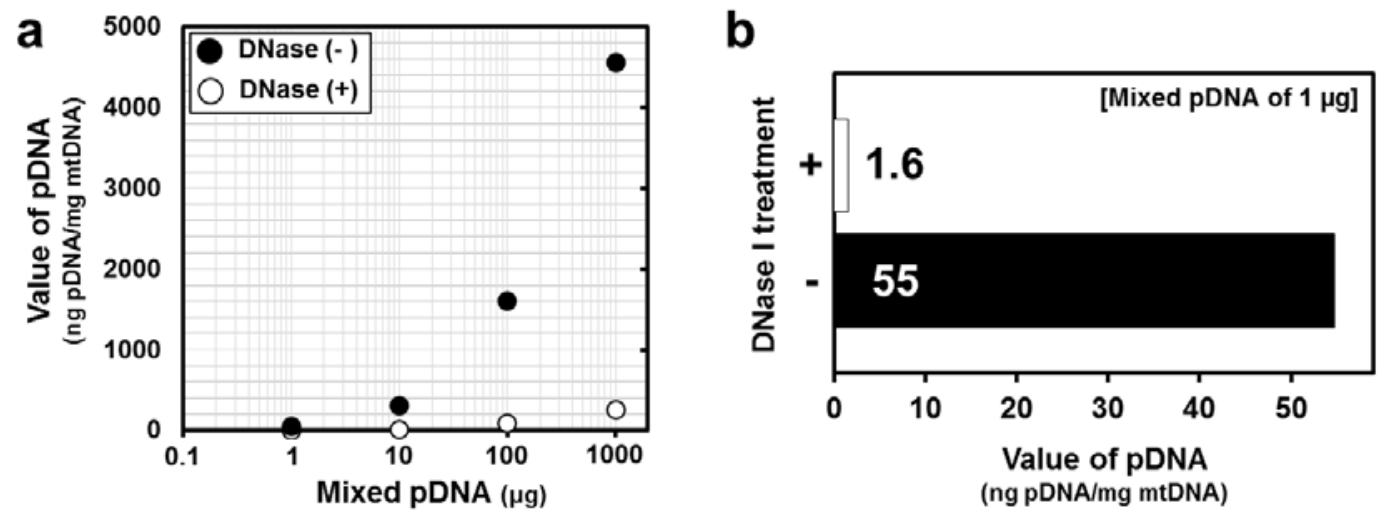

(a) Various amounts of pDNA were mixed with the mitochondria-enriched fraction, and DNase I treatment was performed. The value of pDNA was then quantified using PCR methods. (b) The value of pDNA before and after DNase I treatment, when $1 \mu \mathrm{g}$ of pDNA was mixed with the mitochondriaenriched fraction. 


\section{Supplementary Table}

Table S1. Primers used for the PCR to quantify pDNA and mtDNA.

Primers used for the PCR to quantitative pDNA and mtDNA

\begin{tabular}{lll}
\hline Primers & Nucleotide sequence & Purpose \\
\hline Luc (+) & 5'-GGTCCTATGATTATGTCCGGTTATG-3' & \\
Luc $(-)$ & 5'-ATGTAGCCATCCATCCTTGTCAAT-3' & \\
Rat D-loop (+) & 5'-GCTTCTTCGCATTCCACTTC-3' & \\
Rat D-loop (-) & 5'-TCGGAGTTTAATCCTGTGGG-3' & \\
\hline
\end{tabular}

PCR assays to detect the D-loop and luciferase genes were performed in order to detect endogenous mtDNA and exogenous pDNA, respectively. Luc, luciferase; Rat D-loop, rattus mitochondrial displacement loop. 